\title{
Ki67 proliferative index of the neuroendocrine component drives MANEC prognosis
}

\author{
Massimo Milione1, Patrick Maisonneuve², Alessio Pellegrinelli', Federica Grillo3, Luca Albarello4, \\ Paola Spaggiari5, Alessandro Vanoli6, Giovanna Tagliabue7, Eleonora Pisa, Luca Messerini9, \\ Giovanni Centonze10,11, Frediano Inzani12, Aldo Scarpa13, Mauro Papotti14, Marco Volante14, Fausto Sessa15, \\ Nicola Fazio16, Giancarlo Pruneri117, Guido Rindi18, Enrico Solcia6, Stefano La Rosa19 and Carlo Capella15
}

\author{
11st Pathology Division, Department of Pathology and Laboratory Medicine, Fondazione IRCCS Istituto Nazionale dei Tumori, Milan, Italy \\ 2Division of Epidemiology and Biostatistics, European Institute of Oncology (IEO), Milan, Italy \\ ${ }^{3}$ Pathology Unit, Department of Surgical Sciences and Integrated Diagnostics (DISC), University of Genova and Policlinico San Martino, Genova, Italy \\ 4Pathology Unit, IRCCS San Raffaele Scientifica Institute, Milan, Italy \\ ${ }^{5}$ Cancer Center Humanitas, Milan, Italy \\ ${ }^{6}$ Fondazione IRCCS Policlinico San Matteo and Department of Molecular Medicine, University of Pavia, Pavia, Italy \\ 'Lombardy Cancer Registry, Varese Province Cancer Registry Unit, Department of Research, Fondazione IRCCS Istituto Nazionale dei Tumori, Milan, Italy \\ ${ }^{8}$ Division of Pathology, European Institute of Oncology (IEO), Milan, Italy \\ 'Diagnostic and Molecular Pathology, Department of Experimental and Clinical Medicine, University of Florence, Firenze, Italy \\ ${ }^{10}$ Department of Experimental Oncology and Molecular Medicine, Unit of Tumor Genomics, Fondazione IRCCS Istituto Nazionale dei Tumori, Milan, Italy \\ ${ }^{11}$ Clinical Research Lab (CRAB), Department of Pathology and Laboratory Medicine, Fondazione IRCCS Istituto Nazionale dei Tumori, Milan, Italy \\ ${ }^{12}$ Anatomic Pathology Unit, Fondazione Policlinico Universitario A. Gemelli, Rome, Italy \\ ${ }^{13}$ ARC-Net Research Centre and Department of Diagnostics and Public Health-Section of Pathology, University and Hospital Trust of Verona, Verona, Italy \\ 14Department of Oncology, University of Turin, Turin, Italy \\ ${ }^{15}$ Department of Medicine and Surgery, University of Insubria, Varese, Italy \\ ${ }^{16}$ Gastrointestinal Medical Oncology and Neuroendocrine Tumors Unit, European Institute of Oncology (IEO), Milan, Italy \\ 17 University of Milan, School of Medicine, Milan, Italy \\ ${ }^{18}$ Institute of Anatomic Pathology, Università Cattolica del Sacro Cuore-Fondazione Policlinico Universitario A. Gemelli, Rome, Italy \\ ${ }^{19}$ Service of Clinical Pathology, Lausanne University Hospital, Institute of Pathology, Lausanne, Switzerland
}

Correspondence should be addressed to M Milione: massimo.milione@istitutotumori.mi.it

\begin{abstract}
Mixed adenoneuroendocrine carcinomas (MANECs) are composed of a poorly differentiated neuroendocrine carcinoma (NEC) and a non-neuroendocrine (non-NEC) neoplastic epithelial component, each representing at least $30 \%$ of the tumor. At present, prognostic factors for MANECs remain largely unexplored. We investigated the clinical-pathologic features of a large multicenter series of digestive system MANECs. Surgical specimens of 200 MANEC candidates were centrally reviewed; diagnosis was confirmed in 160 cases. While morphology, proliferation (mitotic count (MC), Ki67 index) and immunophenotype (p53, SSTR2a, beta-Catenin, Bcl-2, p16, Rb1, ALDH, mismatch repair proteins and $C D 117)$ were investigated separately in both components, genomic (TP53, KRAS, BRAF) alterations were searched for on the entire tumor. Data were correlated with overall survival (OS). MANEC sites were: 92 colorectal, 44 gastroesophageal and 24 pancreatobiliary. Median OS was 13.2 months. After adjustment for primary site, Ki67 index of the NEC component (but not of the non-NEC component) was the most powerful prognostic marker. At multivariable analysis, patients with $\mathrm{Ki} 67 \geq 55 \%$ had an 8 -fold risk of death (hazard ratio (HR) 7.83; $95 \%$ confidence interval (CI) 4.17-14.7; $P<0.0001$ ) and a median OS of 12.2 months compared to those with Ki67 $<55 \%$ (median OS 40.5 months). MC (HR $1.51 ; 95 \% \mathrm{Cl} 1.03-2.20, P=0.04$ ) was a
\end{abstract}

\author{
Key Words \\ - MANEC \\ - NEC \\ - Ki67 \\ - prognosis
}


weaker prognostic index. Colorectal primary site (HR 1.60; $95 \% \mathrm{Cl} 1.11-2.32 ; P=0.01$ ) was significantly associated with poorer survival. No single immunomarker, in either component, was statistically significant. This retrospective analysis of a large series of digestive system MANECs, showed that the NEC component, particularly its Ki67 index, was the main prognostic driver.

\section{Introduction}

The coexistence of neuroendocrine and nonneuroendocrine components in the same neoplasm is a rare but well-known phenomenon in digestive system tumors. The 2010 World Health Organization (WHO) classification of tumors of the digestive system proposed the term 'mixed adenoneuroendocrine carcinoma' (MANEC) to define these cancers in which, by definition, each component represents at least 30\% of the tumor mass (Bosman et al. 2010). The pathogenesis of MANECs is still unclear, and different hypotheses have been proposed to explain their biphasic morphology. Molecular investigations have suggested a multistep progression from a common precursor lesion. Indeed, the higher frequency of chromosomal and gene abnormalities found in the neuroendocrine component compared to the nonneuroendocrine component suggests that progression from a non-neuroendocrine toward a neuroendocrine cell phenotype, and not vice-versa, is more frequent (Vortmeyer et al. 1997, Huang et al. 2002, Kim et al. 2002, Furlan et al. 2003, Paniz Mondolfi et al. 2011, Scardoni et al. 2014, Volante et al. 2015, Jesinghaus et al. 2017, Woischke et al. 2017).

The clinical behavior of MANECs is generally aggressive; however, prognostic markers predictive of MANEC outcome have not been definitively validated to date. Whether prognostic markers of poorly differentiated neuroendocrine carcinomas (NECs), such as proliferation, CD117 expression and microsatellite instability, have the same prognostic relevance in MANECs is, as yet, unknown. Moreover, considering the peculiar morphologic characteristics of MANECs, additional specific parameters may be prognostically important, including the percentage and type of different tumor components, tumor site, morphologic features (i.e. tumor cell budding, vascular and perineural infiltration, intra- and peritumoral lymphoid infiltration), type of tumor component in nodal or distant metastases, immunophenotype and molecular profile. To the best of our knowledge, these diverse characteristics have never been fully investigated in a large series of digestive MANECs.
In this retrospective multicenter study, we collected a large series of MANECs and we extensively investigated clinical, morphologic, immunohistochemical and molecular features in order to characterize the different tumor components and to search for parameters useful in prognostic stratification.

\section{Materials and methods}

\section{Case selection and study design}

Between 1995 and 2015, the surgical pathology and clinical databases of eleven Italian institutions were retrospectively searched and patients with one of the following diagnoses at histology report sign out were selected: 'mixed exocrine-neuroendocrine carcinoma', 'adenoneuroendocrine carcinoma (MANEC)', 'composite glandular endocrine carcinoma', 'carcinoma with neuroendocrine differentiation', 'amphicrine/combined, carcinoma or tumor', 'mixed adenocarcinoma and neuroendocrine carcinoma'. Exclusion criteria were (i) cases with only biopsy material available; (ii) cases with either NEC or non-NEC component $<30 \%$; (iii) cases in which the neuroendocrine component was well differentiated (as discussed by La Rosa et al. 2012c, Ohike 2017) and (iv) patients who underwent neoadjuvant chemotherapy.

A total of 200 candidate cases were identified. Patients' charts and tumor morphology were carefully revised, first by the pathologist of the case-proposing hospital and then by a panel of seven expert pathologists (M M, A P, P S, A V, L A, S L R and C C) using a multihead microscope. During panel consensus meetings, the original diagnosis was reviewed and further workup was carried out whenever panelists disagreed or quantitative evaluations approached cut-off values. For qualitative parameters a majority decision was adopted, while for quantitative evaluations, the mean of values obtained by the individual panelists was taken as final. MANEC identification, quantitative evaluation of the NEC vs non-NEC components and subtype characterization as collision or combined 
were based on parallel investigation of at least two consecutive sections from representative blocks, stained with hematoxylin-eosin and synaptophysin, respectively. Ki67 proliferative rate (or other histochemical parameters investigated) was assessed on a third consecutive section. The identification of an amphicrine component was based on finding synaptophysin reactivity within the cytoplasm of cells also showing signet ring or gland-forming patterns after alcian blue counterstaining of the same section or with the help of an adjacent section stained with PAS and alcian blue. In the end, 160 cases met all the above criteria and were enrolled in the study.

Morphologic analysis (Table 1) considered: (a) assessment of the percentage of NEC and non-NEC components; (b) morphology of non-NEC component: adenoma, adenocarcinoma, mucinous carcinoma, signet ring carcinoma, squamous cell carcinoma and acinar cell carcinoma (only in pancreatic site) (La Rosa et al. 2012a); (c) morphology of NEC component: small cell or large cell according to WHO 2010 (Bosman et al. 2010, Rindi et al. 2010); (d) necrosis in the NEC component; (e) Ki67 index was defined using the MIB antibody as a percentage of 500-2000 cells counted in areas of strongest nuclear labeling ('hot spots') (Rindi et al. 2010); (f) mitotic count (MC) was evaluated in at least $10 \mathrm{HPF}\left(10 \mathrm{HPF}=2 \mathrm{~mm}^{2}\right)$ (Rindi et al. 2010); (g) quantitative assessment of NEC, non-NEC or mixed type components in lymph node metastases and/or in distant metastases; (h) tumor staging according to the Union for International Cancer Control/American Joint Committee on Cancer (UICC/AJCC) 8th edition (Amin 2017); (i) lymphovascular invasion (evaluated on both hematoxylin-eosin (H\&E) and/or CD31-stained sections); (l) perineural invasion; (m) intra and/or peritumoral lymphocytic infiltration; (n) prevalent tumor component (NEC, non-NEC or mixed type) on the deep invasive front; (o) type of combination of the NEC and non-NEC component: 'collision' when the two components were clearly demarcated, 'combined' when they were intimately admixed and 'amphicrine/combined', when the same cells displayed both neuroendocrine and non-neuroendocrine phenotype (as a rule this was observed in a combined histological background and (p) tumor budding defined according to the International Tumor Budding Consensus Conference 2016 (ITBCC) (Lugli et al. 2017).

The histochemical and immunohistochemical (IHC) study (Supplementary Table 1, see section on supplementary data given at theend of this article) included: (a) Alcian Blue-Periodic Acid-Schiff (PAS) to better define mucin production in the non-NEC epithelial neoplastic component; (b) synaptophysin and chromogranin-A (general neuroendocrine immunomarkers) in order to confirm the presence and extent of the NEC component (Fig. 1B); (c) Ki67 staining evaluated in both NEC and nonNEC components (Bosman et al. 2010, Rindi et al. 2010); (d) IHC assessment and evaluation in both components of several markers including p53, Rb1, p16, Bcl-2, Betacatenin, aldehyde dehydrogenase (ALDH), CDX2, thyroid transcription factor-1 (TTF1), mismatch repair (MMR) proteins, CD117 and somatostatin receptor 2A (SSTR2A) using the antibodies listed in Supplementary Table 2 .

With the exception of p53 and SSTR2A, all markers were considered positive regardless of the number of positive cells. p53 was considered positive when $\geq 30 \%$ of cells were positive (Ali et al. 2017); SSTR2A was assessed according to Volante et al. (positive: 2+, 3+; negative: 0, 1+ score) (Volante et al. 2007). MMR deficiency was established according to the criteria reported by Chiaravalli et al. (2001).

Data concerning mutations (Table 1) of KRAS (codons 12 and 13), BRAF (codon 600) and TP53 (exons 5-8) were extracted from investigations performed for therapy decision making, either by PCR pyrosequencing as described by (Sahnane et al. 2015) or by next-generation sequencing analysis (NGS) as detailed by (Meazza et al. 2016).

This study was performed according to the clinical standards of the 1975 and 1983 Declaration of Helsinki and was approved by the Ethical Committee of Fondazione IRCCS Istituto Nazionale dei Tumori, Milan, Italy $\left(\mathrm{n}^{\circ}\right.$ INT 21/16).

\section{Evaluation of proliferative cut-offs}

We searched for prognostically relevant cut-offs of Ki67 and MC. The results of our exploratory analysis are shown in Supplementary Fig. 1.

\section{Statistical analysis}

Data were analyzed by descriptive statistics. Differences in frequencies were assessed with the chi-square or the Fisher exact test. The primary study endpoint was the correlation of overall survival (OS) with primary tumor site, tumor stage, NEC subtype (large or small cell), non-NEC histotype, MANEC type (collision, combined, amphicrine/combined), percentage of NEC and non-NEC components (evaluated on: whole neoplasm, invasive front, lymph nodes and/or distant metastases), lymphocytic intratumoral and peritumoral infiltrate, angioinvasion, perineural invasion and necrosis in the NEC component. The following parameters were separately evaluated in NEC and 
Table 1 Main characteristics of patients with MANEC according to tumor site.

\begin{tabular}{|c|c|c|c|c|c|}
\hline & All & Colorectal & Gastroesophageal & Pancreatobiliary & $P$-value \\
\hline Total & 160 & 92 & 44 & 24 & \\
\hline \multicolumn{6}{|l|}{ Sex } \\
\hline Male & 116 & 61 & 38 & 17 & \\
\hline Female & 44 & 31 & 6 & 7 & 0.04 \\
\hline \multicolumn{6}{|l|}{ Age } \\
\hline$<60$ & 40 & 25 & 12 & 3 & \\
\hline $60-69$ & 55 & 31 & 14 & 10 & \\
\hline $70+$ & 65 & 36 & 18 & 11 & 0.66 \\
\hline \multicolumn{6}{|l|}{ Stage } \\
\hline $\mathrm{I} / \mathrm{II}(\mathrm{pN}-)$ & 11 & 3 & 3 & 5 & \\
\hline IIIA (pN-) & 13 & 6 & 1 & 6 & \\
\hline IIIB $(p N+)$ & 119 & 74 & 34 & 11 & \\
\hline IV $(\mathrm{M}+)$ & 17 & 9 & 6 & 2 & 0.002 \\
\hline \multicolumn{6}{|l|}{ MANEC subtype } \\
\hline Collision & 54 & 35 & 12 & 7 & \\
\hline Combined & 82 & 44 & 26 & 12 & \\
\hline Amphicrine/Combined & 24 & 13 & 6 & 5 & 0.63 \\
\hline \multicolumn{6}{|l|}{ NEC type } \\
\hline Large cells & 135 & 78 & 41 & 16 & \\
\hline Small cells & 25 & 14 & 3 & 8 & 0.02 \\
\hline \multicolumn{6}{|l|}{ NEC necrosis } \\
\hline Absent & 40 & 23 & 8 & 9 & \\
\hline Present & 120 & 69 & 36 & 15 & 0.21 \\
\hline \multicolumn{6}{|l|}{$\%$ NEC component } \\
\hline$<50 \%$ & 39 & 19 & 14 & 6 & \\
\hline $50-60 \%$ & 68 & 40 & 18 & 10 & \\
\hline$>60 \%$ & 53 & 33 & 12 & 8 & 0.70 \\
\hline \multicolumn{6}{|l|}{ Ki67 (NEC) } \\
\hline$<55 \%$ & 28 & 13 & 7 & 8 & \\
\hline$\geq 55 \%$ & 132 & 79 & 37 & 16 & 0.10 \\
\hline \multicolumn{6}{|l|}{ Ki67 (non-NEC) } \\
\hline$<55 \%$ & 77 & 47 & 17 & 13 & \\
\hline$\geq 55 \%$ & 83 & 45 & 27 & 11 & 0.34 \\
\hline \multicolumn{6}{|l|}{ MC (NEC) } \\
\hline$<50 / 10 \mathrm{HPF}$ & 111 & 62 & 32 & 17 & \\
\hline$\geq 50 / 10 \mathrm{HPF}$ & 49 & 30 & 12 & 7 & 0.81 \\
\hline \multicolumn{6}{|l|}{$M C$ (non-NEC) } \\
\hline$<50 / 10 \mathrm{HPF}$ & 132 & 73 & 39 & 20 & \\
\hline$\geq 50 / 10 \mathrm{HPF}$ & 28 & 19 & 5 & 4 & 0.41 \\
\hline \multicolumn{6}{|l|}{ Nerve infiltration } \\
\hline Absent & 20 & 12 & 4 & 4 & \\
\hline Present & 106 & 63 & 28 & 15 & 0.69 \\
\hline \multicolumn{6}{|l|}{ Angioinvasion } \\
\hline Absent & 23 & 14 & 6 & 3 & \\
\hline Present & 137 & 78 & 38 & 21 & 1.00 \\
\hline \multicolumn{6}{|l|}{ Budding } \\
\hline Low & 47 & 24 & 17 & 6 & \\
\hline Intermediate & 47 & 33 & 8 & 6 & \\
\hline High & 66 & 35 & 19 & 12 & 0.21 \\
\hline \multicolumn{6}{|l|}{ Peritumoral lymphoid cells } \\
\hline Absent & 70 & 42 & 18 & 10 & \\
\hline Mild & 64 & 32 & 18 & 14 & \\
\hline Moderate & 23 & 16 & 7 & 0 & \\
\hline Severe & 3 & 2 & 1 & 0 & 0.19 \\
\hline Intratumoral lymphoid cells & & & & & \\
\hline Absent & 74 & 42 & 22 & 10 & \\
\hline Mild & 69 & 41 & 15 & 13 & \\
\hline Moderate & 13 & 6 & 6 & 1 & \\
\hline Severe & 4 & 3 & 1 & 0 & 0.61 \\
\hline & & & & & (Continued \\
\hline $\begin{array}{l}\text { http://erc.endocrinology-journals.org } \\
\text { https://doi.org/10.1530/ERC-17-0557 }\end{array}$ & $\begin{array}{l}018 \text { Socie } \\
\text { ublished } \\
\operatorname{Pr}\end{array}$ & $\begin{array}{l}\text { rinology } \\
\text { fica Ltd. } \\
\text { t Britain }\end{array}$ & & & \\
\hline
\end{tabular}


Table 1 Continued

\begin{tabular}{l}
\hline N+ (\%NEC) \\
Median (range) \\
$<100 \%$ (Mixed) \\
$100 \%$ (pure NEC) \\
M+ $\%$ NEC) \\
Median (range) \\
$<100 \%$ (Mixed) \\
$100 \%$ (pure NEC) \\
Molecular analysis \\
Wild type \\
KRAS mutated \\
BRAF mutated \\
TP53 mutated
\end{tabular}

\begin{tabular}{c}
\hline All \\
\hline $70(0-100)$ \\
97 \\
35 \\
$85(20-100)$ \\
11 \\
6 \\
38 \\
12 \\
4 \\
17
\end{tabular}

\begin{tabular}{|l|}
\hline Colorectal \\
$\begin{array}{l}70(0-100) \\
57 \\
23\end{array}$
\end{tabular}

\begin{tabular}{c}
\hline Gastroesophageal \\
\hline $70(20-100)$ \\
31 \\
8
\end{tabular}

\begin{tabular}{c} 
Pancreatobiliary \\
\hline $90(0-100)$ \\
9 \\
4
\end{tabular}

$90(20-100)$

5

4

55 (20-100)

4

2

$88(85-90)$

2

0

0.80

*Some immunohistochemical evaluations are missing for some patients. Budding: single tumor cell or a cell cluster (buds) of up to 4 tumor cells, detected at the invasive tumor front; Low grade budding: <4 buds; Intermediate grade budding: $5-10$ buds; High-grade budding: $>10$ buds. Ki67, Ki67 index; M+, Liver metastasis; MANEC, Mixed adenoneuroendocrine carcinomas; MC, Mitotic count; N+, Lymph node metastasis; NEC, neuroendocrine carcinoma.

non-NEC components: MC, Ki67, MMR deficiency, Bcl-2, Rb1, p16, p53, TTF1, CDX2, CD117, ALDH, beta-catenin and SSTR2a.

OS was assessed from the time of diagnosis to the time of death or last follow-up. Survival curves were drawn according to the Kaplan-Meier method, and difference between groups was assessed with the log rank test. The proportions of patients surviving at different time points are presented with respective 95\% CI. Univariable and multivariable Cox proportional hazards regression analysis was used to assess the prognostic significance of various clinical and histopathologic characteristics. Data analysis was performed using the SAS software (version 9.4, Cary, NC, USA). All tests were two sided and $P$ values $<0.05$ were considered statistically significant.

\section{Result's}

\section{Clinicopathologic features}

Figure 1 and Table 1 summarize the main clinicopathologic features of the 160 patients enrolled in the study. The series comprised more males than females (72.5\% vs $27.5 \%)$, and this difference was statistically significant and was maintained across tumor sites $(P=0.04)$. Most patients (74.4\%) had stage IIIB disease. MANECs were more frequently colorectal $(n=92,57.5 \%)$, with a prevalence in the right colon (64 cases) vs the left colon (10 cases) and rectum (18 cases), followed by gastroesophageal ( $n=44$, $27.5 \%$ ) locations, with a prevalence (32 cases) for stomach vs cardio-esophageal junction (9 cases) and esophageal tumors (3 cases). Finally, pancreatobiliary locations were the least common $(n=24,15.0 \%)$, the majority of these were pancreatic tumors (14 cases) compared to gallbladder (7 cases) or extrahepatic biliary tract primaries (3 cases). No MANECs were found in the small bowel or appendix.

All neoplasms investigated showed $30 \%$ or more reactive cells for synaptophysin, while only 90/160 cases showed significant reactivity for chromogranin-A. Thus, we based our identification of the NEC component on the synaptophysin-positive, poorly differentiated neuroendocrine part of the neoplasm (Fig. 1A and B). Most MANECs (75.6\%) had a NEC component in the 50-70\% range (Table 1). Considering the NEC component, the large cell type ( $n=135,84.4 \%)$ was more frequent compared to the small cell type. In the non-NEC component, conventional adenocarcinoma was the dominant histotype $(n=105)$, followed by mucinous ( $n=17,11$ of which colorectal), signet ring cell ( $n=10,8$ of which gastric) and squamous cell carcinoma ( $n=5,4$ of which esophageal or cardioesophageal). Fifty-four (33.8\%) tumors showed a 'collision' pattern, $82(51.2 \%)$ a purely 'combined' pattern, and 24 (15.0\%) a mixed 'amphicrine' and combined pattern. In 'collision' neoplasms, the adenocarcinomatous component was usually overlying a deep, invasive NEC component. In 'combined' neoplasms, the two components were admixed without distinction between superficial and deep aspects (Fig. 1A and B); however, the invasive tumor front (Fig. 1C) was predominantly of NEC type with a pure NEC component in $71(44.4 \%)$ cases, mixed NEC/non-NEC component in $79(49.4 \%)$ cases and pure non-NEC carcinomatous component in 10 cases (6.2\%) only. Angioinvasion was seen in all MANEC cases. Neoplastic emboli in vessels were prevalently of NEC or mixed NEC/non-NEC type. 

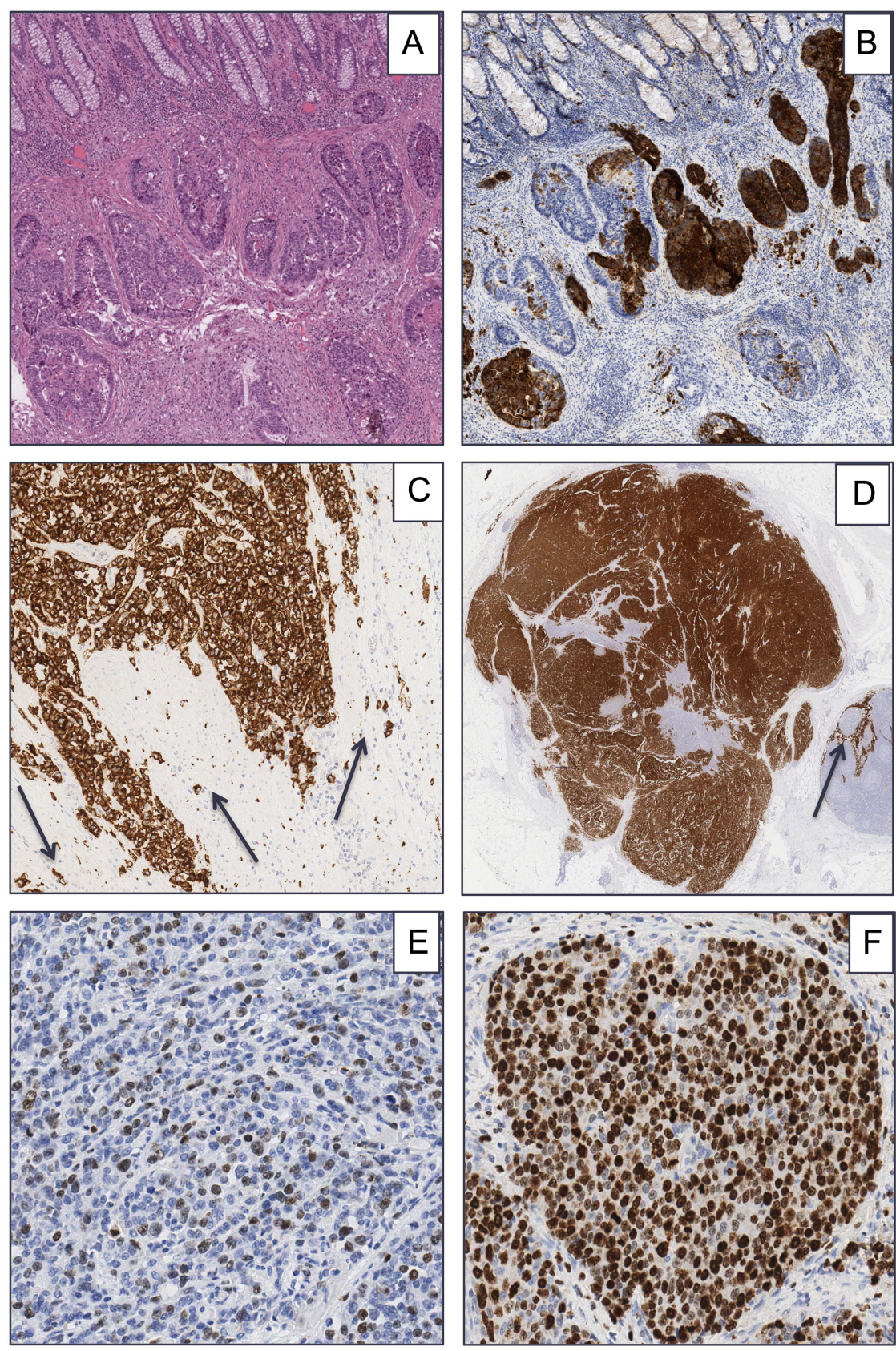

\section{Figure 1}

Two adjacent sections of a combined MANEC illustrate the intimate admixture of the NEC and non-NEC components of the neoplasm. (A) Hematoxylin/Eosin; (B) Synaptophysin Immunohistochemistry. Both $\times 100$; (C) Invasive front of colonic MANEC showing exclusively a NEC component massively reactive for Synaptophysin. Note the associated high-grade budding (arrows) $\times 100$; (D) Synaptophysin immunostained intestinal MANEC in lymph node metastases, massive in the left lymph node and sinusoidal only (arrow) in the right lymph node, both composed exclusively of NEC cells. $\times 100$. (E) NEC component of a MANEC with $40 \%$ of Ki67 proliferative index. $\times 400$; (F) NEC component of a MANEC with $90 \%$ of Ki67 index $\times 400$.
Nodal metastases (Fig. 1D) were of pure NEC histotype in 35 (26.5\%) and mixed NEC/non-NEC in 97 (73.5\%) cases. In distant metastases, pure NEC histology was found in 6 (35.3\%) cases, and mixed NEC/non-NEC histology in 11 cases (64.7\%). Pure non-NEC component was not detected in metastases.

\section{Survival analysis}

In the overall cohort, median OS was 13.2 months (95\% CI 12.4-14.3) (Fig. 2A).

\section{Site}

OS was significantly shorter in patients with colorectal tumors (median 12.2 months; 95\% CI 10.3-13.3) compared to patients with tumors in other digestive system sites: gastroesophageal or pancreatobiliary (median 17.3 months; 95\% CI 13.3-19.9) ( $P=0.001)$ (Fig. 2B).

\section{Immunohistochemistry markers}

The majority of IHC markers showed no statistical association with OS (Supplementary Table 1). Only CD117 (Table 2), evaluated in the NEC component, was 
A

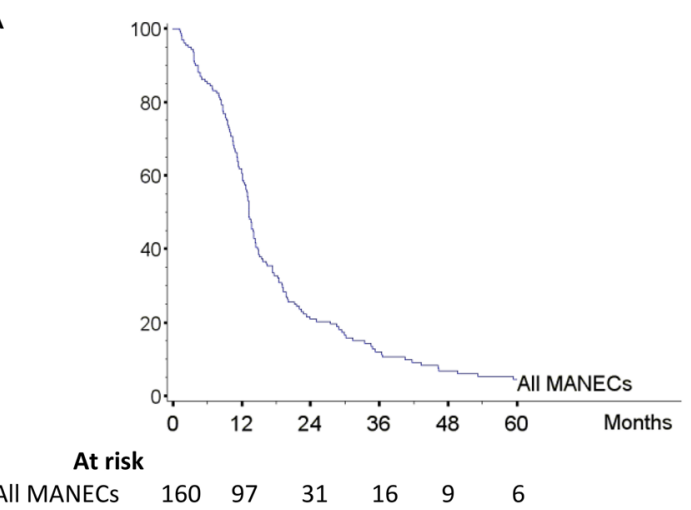

C

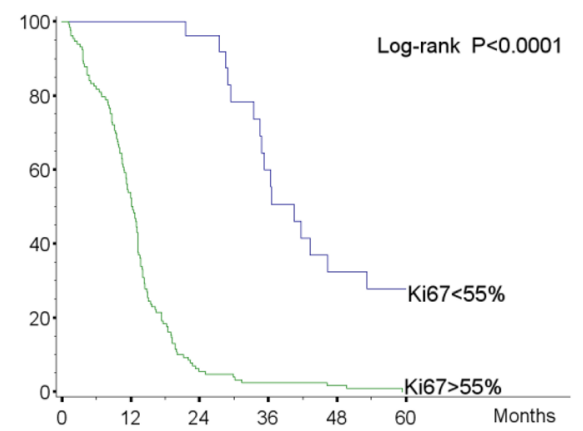

$\begin{array}{cllllll}\text { At risk } & & & & \\ \mathrm{Ki} 67<55 \% & 28 & 28 & 24 & 13 & 7 & 6\end{array}$
B

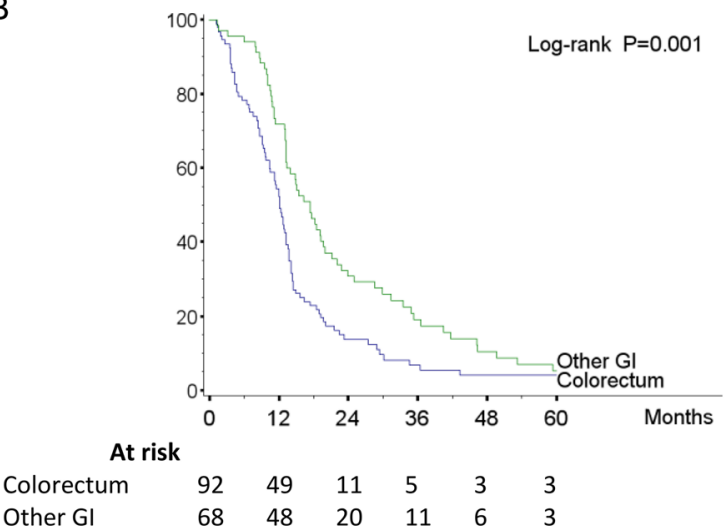

D

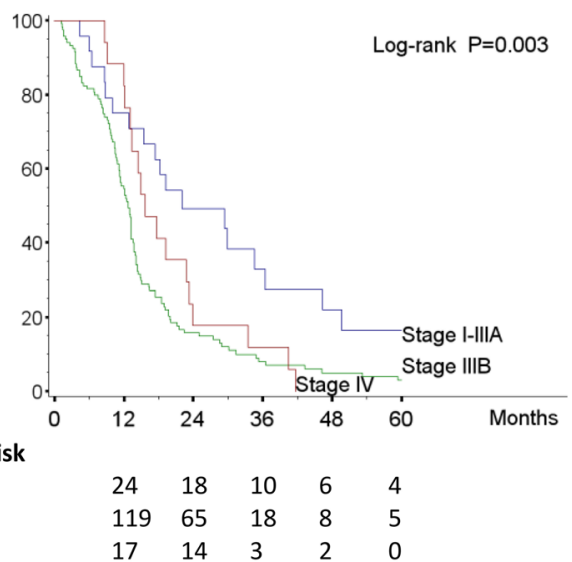

Stage I-IIIA

Stage IIIB

Stage IV

\section{Figure 2}

Overall survival of the whole 160 MANECs population (A), of MANECs according to tumor site (B) or according to Ki67 proliferative index (C) or according to AJCC staging system (D). A full color version of this figure is available at https://doi.org/10.1530/ERC-17-0557.

associated with $\mathrm{OS}$ at univariable analysis $(\mathrm{HR}=1.59 ; 95 \%$ CI 1.13-2.24; $P=0.008)$, although the association lost statistical significance at multivariable analysis $(\mathrm{HR}=1.42$; 95\% CI 0.99-2.04; $P=0.06$ ). Loss of MMR proteins (MLH1 and PMS2 in all cases) was found in 8/160 neoplasms with equal involvement of NEC and non-NEC components and no significant influence on OS (Supplementary Table 1). Rb, p53, CD117, CDX2 and ALDH were significantly co-expressed in both NEC and non-NEC components (Supplementary Table 3). The distribution of immunohistochemical markers in the NEC and non-NEC components is reported in Supplementary Table 1.

\section{Proliferation}

A preliminary evaluation of the NEC component showed $55 \%$ to be an optimal prognostic cut-off for Ki67 index and 50 mitoses/10 HPF to be optimal for MC (Supplementary Fig. 1). Ki67 index turned out to be $\geq 55 \%$ in the large majority (82.5\%) of cases, while MC was $\geq 50$ in $30.6 \%$ of cases. On the other hand, in the non-NEC component,
Ki67 was $\geq 55 \%$ in $51.9 \%$ and MC was $\geq 50 / 10$ HPF in $17.5 \%$ of cases.

Patients with Ki67 index $<55 \%$ in the NEC component (median 40.5 months; $95 \%$ CI 34.5-53.2) had a significatively longer OS than those with $\mathrm{Ki} 67 \geq 55 \%$ (median 12.2 months; 95\% CI 11.1-13.2) $P<0.0001$. The latter showed a hazard ratio (HR) of 9.08 (95\% CI $5.13-16.1)$ vs $\mathrm{Ki} 67<55 \%(P<0.0001)$ after adjustment for tumor site, which retained high significance at multivariable analysis (Fig. 2C and Table 2). Patients with $\mathrm{MC}<50$ mitoses/10 HPF also had significatively longer OS (median 14.1; 95\% CI 13.0-16.4) compared to those with $\geq 50$ mitoses/10 HPF (median 11.2 months; 95\% CI 9.5-13.2; $P=0.002$ ), although with clearly lower HR (Table 2). In the non-NEC component, a Ki67 index of $\geq 55 \%$ was associatewd with a tumor site-adjusted HR of $1.81 ; 95 \%$ CI $1.28-2.54(P=0.0007)$ vs $<55 \%$, which lost statistical significance at multivariable analysis, while a MC $\geq 50 / 10$ HPF lacked any significant difference compared to $\mathrm{MC}<50 / 10 \mathrm{HPF}$. 
Table 2 Univariate and multivariable analysis for overall survival.

\begin{tabular}{|c|c|c|c|c|c|c|}
\hline & $\begin{array}{l}\text { Adjusted for site } \\
\text { HR }(95 \% \mathrm{Cl}) *\end{array}$ & P-value & $\begin{array}{c}\text { Multivariable } 2 \text { HR } \\
(95 \% \mathrm{Cl})\end{array}$ & $P$-value & $\begin{array}{c}\text { Multivariable } 3 \text { HR } \\
(95 \% \mathrm{Cl})\end{array}$ & P-value \\
\hline \multicolumn{7}{|l|}{ Site } \\
\hline Colorectal & 1.00 & & 1.00 & & 1.00 & \\
\hline Gastroesophageal & $0.73(0.50-1.06)$ & 0.10 & $0.61(0.39-0.94)$ & 0.02 & $0.64(0.43-0.96)$ & 0.03 \\
\hline Pancreatobiliary & $0.41(0.25-0.67)$ & 0.0004 & $0.54(0.29-1.03)$ & 0.06 & $0.58(0.33-1.04)$ & 0.07 \\
\hline \multicolumn{7}{|l|}{ Stage } \\
\hline I/II/IIIA (pN-) & 1.00 & & 1.00 & & 1.00 & \\
\hline IIIB (pN+) & $2.03(1.20-3.42)$ & 0.008 & $1.70(0.90-3.22)$ & 0.10 & $1.75(0.95-3.23)$ & 0.07 \\
\hline IV (M+) & $1.59(0.80-3.16)$ & 0.19 & $1.08(0.48-2.40)$ & 0.86 & $1.09(0.51-2.31)$ & 0.83 \\
\hline \multicolumn{7}{|l|}{ MANEC subtype } \\
\hline Collision & 1.00 & & 1.00 & & & \\
\hline Combined & $0.90(0.63-1.28)$ & 0.56 & $1.07(0.72-1.61)$ & 0.73 & - & \\
\hline Amphicrin/Combined & $0.56(0.33-0.96)$ & 0.04 & $0.83(0.45-1.53)$ & 0.55 & - & \\
\hline \multicolumn{7}{|l|}{ NEC type } \\
\hline Large cells & 1.00 & & 1.00 & & & \\
\hline Small cells & $1.54(0.97-2.43)$ & 0.07 & $1.27(0.77-2.10)$ & 0.35 & - & \\
\hline \multicolumn{7}{|l|}{ NEC component } \\
\hline$\%$ NEC component (per 10\%) & $1.09(0.96-1.22)$ & 0.18 & - & & - & \\
\hline Ki67 ( $\geq 55 \%$ vs <55\%) & $9.08(5.13-16.1)$ & $<0.0001$ & $8.92(3.96-20.1)$ & $<0.0001$ & $7.83(4.17-14.7)$ & $<0.0001$ \\
\hline MC ( $\geq 50 / 10$ HPF vs <50/10 HPF) & $1.81(1.28-2.58)$ & 0.0009 & $1.53(1.03-2.28)$ & 0.04 & $1.51(1.03-2.20)$ & 0.04 \\
\hline p53 ( $\geq 30 \%$ vs <30\%) & $1.10(0.78-1.56)$ & 0.58 & - & & - & \\
\hline CD117 (positive vs negative) & $1.59(1.13-2.24)$ & 0.008 & $1.45(0.98-2.15)$ & 0.06 & $1.42(0.99-2.04)$ & 0.06 \\
\hline SSTR2a (positive vs negative) & $1.21(0.87-1.68)$ & 0.25 & - & & & \\
\hline \multicolumn{7}{|l|}{ Non-NEC component } \\
\hline Ki67 ( $\geq 55 \%$ vs <55\%) & $1.81(1.28-2.54)$ & 0.0007 & $1.29(0.87-1.92)$ & 0.20 & - & \\
\hline MC ( $\geq 50 / 10$ HPF vs <50/10 HPF) & $1.39(0.91-2.11)$ & 0.13 & - & & - & \\
\hline \multicolumn{7}{|l|}{ Nerve infiltration } \\
\hline Present vs absent & 3.20 (1.77-5.77) & 0.0001 & $0.88(0.42-1.82)$ & 0.72 & - & \\
\hline \multicolumn{7}{|l|}{ Angioinvasion } \\
\hline Present vs absent & $0.85(0.54-1.33)$ & 0.47 & - & & - & \\
\hline \multicolumn{7}{|l|}{ Budding } \\
\hline Absent & 1.00 & & 1.00 & & & \\
\hline Mild/moderate & $0.82(0.53-1.27)$ & 0.37 & $1.29(0.72-2.28)$ & 0.39 & - & \\
\hline Severe & $1.97(1.31-2.96)$ & 0.001 & $1.13(0.65-1.96)$ & 0.67 & - & \\
\hline \multicolumn{7}{|l|}{ Molecular analysis** } \\
\hline Wild type & 1.00 & & 1.00 & & 1.00 & \\
\hline Mutated & 2.36 (1.41-3.97) & 0.001 & 2.22 (1.19-4.15) & 0.01 & $2.68(1.50-4.81)$ & 0.0009 \\
\hline
\end{tabular}

Multivariable model 2 includes all factors associated with overall survival after single adjustment for tumor site; Multivariable model 3 retains only variables showing an association $(P<0.10)$ with overall survival; ** Multivariable risk estimates for single gene mutations from alternative model $3: K R A S$ mutated ( $n=12 ; \mathrm{HR}=2.69$ (1.24-5.82); $P=0.01) ; B R A F$ mutated $(n=4 ; \mathrm{HR}=1.81$ (0.51-6.39); $P=0.36) ; T P 53$ mutated $(n=17 ; \mathrm{HR}=2.90(1.48-5.68) ; P=0.002)$. CD117, tyrosine-protein kinase Kit; Ki67, Ki67 index; M+, Liver metastases; MANEC, Mixed adenoneuroendocrine carcinoma; MC, Mitotic count; N+, Lymph node metastases; NEC, neuroendocrine carcinoma; SSTR2a, somatostatin receptor 2a.

\section{Stage}

Patients with early-stage (I-IIIA) tumor (24 cases) had longer OS (median 22.1 months; 95\% CI 12.8-36.4) compared to 119 patients with stage IIIB (median 12.7 months; $95 \%$ CI 11.2-13.3) or 17 patients with stage IV disease (median 15.7 months; 95\% CI 12.2-23.3), with a significant difference $(P=0.003)$ between I-IIIA and IIIB+IV cases (Fig. 2D).

When an analysis of OS adjusted for tumor site was performed, OS was shown to be significantly associated with tumor stage, MANEC subtype (mixed amphicrine/ combined vs collision), perineural infiltration, high-grade budding (Fig. 1C) and NGS gene mutations, in addition to
Ki67, MC and CD117 in the NEC component, and Ki67 only in the non-NEC component (Table 2).

\section{Multivariable analysis}

At multivariable analysis, only tumor site, Ki67 and MC in the NEC component and mutations were independently associated with OS (Table 2). Patients with gastroesophageal tumors $(\mathrm{HR}=0.64 ; 95 \%$ CI $0.43-0.96 ; \quad P=0.03)$ and those with pancreatobiliary tumors ( $\mathrm{HR}=0.58 ; 95 \%$ CI $0.33-1.04 ; P=0.07$ ) had significantly better survival compared to patients with colorectal MANECs. Patients with $\mathrm{Ki} 67 \geq 55 \%$ in the NEC component had a 7.83 fold 
increased risk of death (95\% CI 4.17-14.7; $P<0.0001$ ) compared to patients with a Ki67<55\%, while patients with $\mathrm{MC} \geq 50$ mitoses/10 HPF had a 1.51 fold risk of death (95\% CI 1.03-2.20, $P=0.04$ ). A mutation in either KRAS or BRAF or TP53 genes (HR for any mutation 2.68 ; $95 \%$ CI $1.50-4.81 ; P=0.0009$ ) was significantly associated with unfavorable outcome (Table 2). The risk was comparable KRAS mutations (HR 2.69; 95\% CI 1.24-5.82; $P=0.01$ ) and TP53 mutations (HR 2.90; 95\% CI 1.48-5.68; $P=0.002$ ) (Footnote Table 2).

\section{Discussion}

This study demonstrates that the prognosis of MANECs from digestive system is driven mostly by the NEC component with special reference to its Ki67 proliferative index. Ki67 index of the NEC component has proven to be far superior to MC or, indeed, to any other prognostic parameter, either morphologic (prevalence of NEC vs non-NEC component, NEC small or large cell histology, histologic type), immunohistochemical (p53, Rb1, Bcl-2, p16, Cdx2, MMR deficiency, TTF1, ALDH, CD117,SSTR2A protein expression or proliferative index of the non-NEC component) or molecular (TP53 or KRAS or BRAF mutation). Of such parameters, only primary site, CD117 immunostaining of the NEC component, perineural infiltration, high-grade budding and TP53 or KRAS mutations were associated with shorter OS at univariate analysis, while only site and mutations (as well the aforementioned NEC component Ki67 index) retained prognostic value at multivariable analysis (Table 2).

Accordingly, in the diagnostic work up of MANECs, besides identifying the NEC component histologically and immunohistochemically (by synaptophysin and chromogranin-A reactivity), evaluation should also focus on the quantitative assessment of Ki67 index in the neuroendocrine component. While proliferative rate has been widely shown to be an important diagnostic tool for neuroendocrine neoplasms, this study, has added new and interesting findings with regards to the importance of proliferation in MANECs as well. Indeed, as previously reported for pure NECs (Sorbye et al. 2013), a Ki67 threshold of 55\% also distinguishes between two different prognostic classes of MANECs with significant survival differences. These findings parallel our previously published results on pure gastroenteropancreatic NECs (Milione et al. 2017) and show that the OS of MANECs with Ki67 $\geq 55 \%$ closely reproduces that of corresponding pure poorly differentiated NECs with Ki67 $\geq 55 \%$ (type C
NECs according to Milione et al. 2017) (Fig. 3B). Conversely, and perhaps more interestingly, with all the limitations of comparing two different studies, MANECs with Ki $67<55 \%$ showed better survival compared to pure poorly differentiated NECs with Ki67 between 21 and 55\% (type B NECs according to (Milione et al. 2017).

Our findings parallel those of a large study on resected colorectal neoplasms, where survival outcomes of NECs were compared with those of patients with high-grade adenocarcinoma (Shafqat et al. 2015): the results demonstrated that the median survival was significantly shorter (7.1 months) for patients with NECs compared with that of patients affected by high-grade adenocarcinoma (36.0 months). Likewise, the 5-year OS of patients with gastric NECs (38.7\%) was poorer than that of patients with gastric adenocarcinoma (51.8\%) (Xie et al. 2017). The frequent finding of the NEC component only at the invasive front of MANECs or in metastases, suggests that this component is mainly responsible for their aggressiveness.

In our study, we showed that the UICC/AJCC staging system developed for site corresponding carcinomas was prognostically informative also for MANECs. Although most patients were diagnosed at an advanced stage (stage IIIB or IV), with severe prognosis, a small proportion (24 cases, 15\%) of potentially curable patients (stage I-IIIA) was identified. As such, our data support the WHO recommendation of considering MANECs as ordinary carcinomas, providing evidence for the application of the relevant dedicated staging in real-life pathology reporting.

The significant association of several molecular markers, including Rb, p16, p53 CDX2, CD117, ALDH and MMR, that we found in both NEC and non-NEC components seems to confirm and to extend previous studies (Furlan et al. 2003, 2013, La Rosa et al. 2012b,c, Jesinghaus et al. 2017, Kloppel 2017, Konukiewitz et al. 2017, Woischke et al. 2017). This finding suggests a possible common origin of the two MANEC components from a pluripotent cancer stem cell, which undergoes divergent differentiation, during tumor progression. The mixed NEC/non-NEC pattern of growth, we found in $73.5 \%$ of nodal and $64.7 \%$ of distant metastases may by itself support the proposed origin of the two components from a single, metastatic, bipotent clone, although the origin of some mixed metastases from cytologically mixed emboli cannot be excluded. Our finding of a greater frequency MANECs in the colon and stomach, where ordinary carcinomas are also highly prevalent, coupled with the apparent lack of MANECs originating in the mesenteric 
A

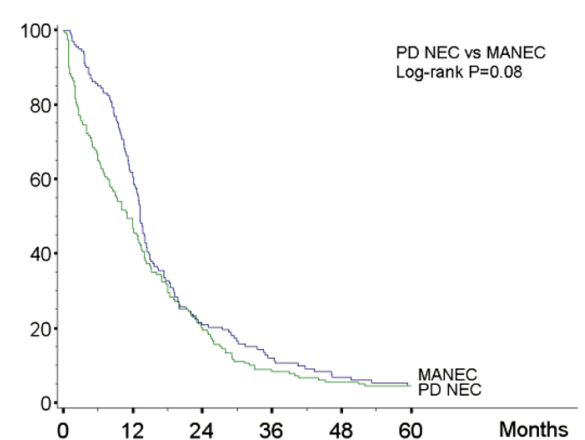

B

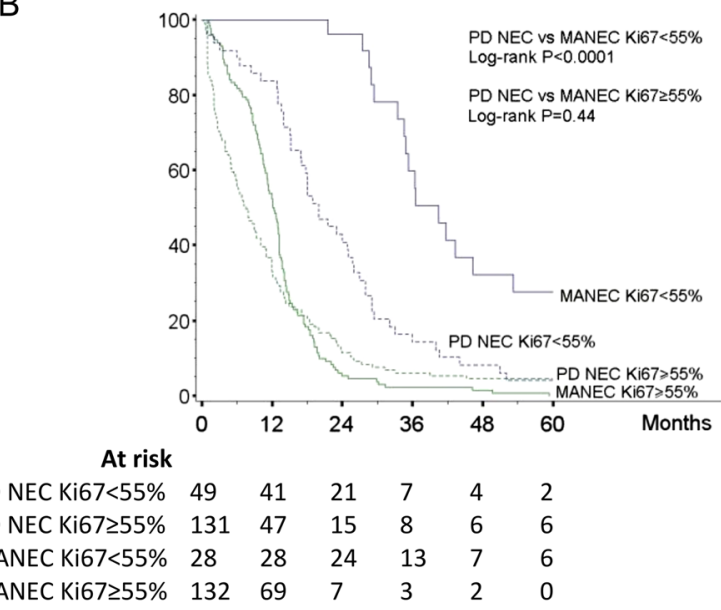

\section{Figure 3}

Comparison between MANECs of current study and pure NECs from our previous study (Milione et al. 2017) (reproduced, with permisison from Milione et al. (2017). Copyright 2017 S. Karger AG, Basel) (A) MANECs overall survival compared to 112 pure poorly differentiated neuroendocrine carcinomas (NECs) one; (B) comparison between type B and C NECs according to Milione et al. (2017) and the present study MANEC subcategories. A full color version of this figure is available at https://doi.org/10.1530/ERC-17-0557.

small intestine and appendix, where conventional carcinomas rarely arise, suggests a MANEC histogenesis more akin to that of classical (adeno)carcinomas than to that of neuroendocrine tumors.

In conclusion, this study of a large series of digestive system MANECs shows that the outcome of these neoplasms is mainly determined by their poorly differentiated neuroendocrine component and that the Ki67 proliferative index (above or below 55\%) is the most important prognostic factor.

Supplementary data

This is linked to the online version of the paper at https://doi.org/10.1530/ ERC-17-0557.

\section{Declaration of interest}

The authors declare no conflicts of interest. Guido Rindi declares that he has received speaker's fee by Novartis Pharma and Ipsen Pharma.

\section{Funding}

Aldo Scarpa is supported by AIRC $5 \times 1000$ n. 18182. Guido Rindi is supported by internal university grants (Università Cattolica line D.1 2015-R412500333 and D.1/2016-R412500403) and by the Associazione Italiana Ricerca sul Cancro - AIRC IG 2013 14696. Institutional support by: Fondazione IRCCS Istituto Nazionale Tumori Milano, Italy; European Institute of Oncology (IEO), Milan, Italy; Fondazione IRCCS Policlinico San Matteo and Department of Molecular Medicine, University of Pavia, Pavia, Italy; IRCCS San Raffaele Scientific Institute, Milan, Italy; Cancer Center Humanitas, Milan, Italy; this research did not receive any specific grant from any funding agency in the public, commercial or not-forprofit sector.

(c) 2018 Society for Endocrinology Published by Bioscientifica Ltd. Printed in Great Britain

\section{Acknowledgments}

The authors thank the following persons for their support: Roberto Buzzoni (Oncology Department, Fondazione IRCCS Istituto Nazionale Tumori, Milan Italy); Adele Busico for NGS study (Biologist at Fondazione IRCCS Istituto Nazionale Tumori, Milan Italy); Carlo Carnaghi (Cancer Center Humanitas, Milan, Italy); The NEXTSTEP multidisciplinary group: Sara Pusceddu (Oncology Department, Fondazione IRCCS Istituto Nazionale Tumori, Milan Italy), Lorenzo Antonuzzo (Università degli Studi, Firenze, Italy), Francesca Spada (IEO, Milan, Italy), Sara Cingarlini (Università degli Studi, Verona, Italy).

\section{References}

Ali AS, Gronberg M, Federspiel B, Scoazec JY, Hjortland GO, Gronbaek H, Ladekarl M, Langer SW, Welin S, Vestermark LW, et al. 2017 Expression of p53 protein in high-grade gastroenteropancreatic neuroendocrine carcinoma. PLoS ONE 12 e0187667. (https://doi. org/10.1371/journal.pone.0187667)

Amin MB 2017 AJCC Cancer Staging Manual 8th Edition. Chicago, IL, USA: AJCC.

Bosman FT, Carneiro F, Hruban R \& Theise N 2010 WHO Classification of Tumours of the Digestive System. Lyon, France: IARC Press.

Chiaravalli AM, Furlan D, Facco C, Tibiletti MG, Dionigi A, Casati B, Albarello L, Riva C \& Capella C 2001 Immunohistochemical pattern of hMSH2/hMLH1 in familial and sporadic colorectal, gastric, endometrial and ovarian carcinomas with instability in microsatellite sequences. Virchows Archiv 438 39-48. (https://doi.org/10.1007/ s004280000325)

Furlan D, Cerutti R, Genasetti A, Pelosi G, Uccella S, La Rosa S \& Capella C 2003 Microallelotyping defines the monoclonal or the polyclonal origin of mixed and collision endocrine-exocrine tumors of the gut. Laboratory Investigation 83 963-971. (https://doi. org/10.1097/01.LAB.0000079006.91414.BE)

Furlan D, Sahnane N, Mazzoni M, Pastorino R, Carnevali I, Stefanoli M, Ferretti A, Chiaravalli AM, La Rosa S \& Capella C 2013 Diagnostic utility of MS-MLPA in DNA methylation profiling of adenocarcinomas and neuroendocrine carcinomas of the colonrectum. Virchows Archiv 462 47-56. (https://doi.org/10.1007/s00428012-1348-2) 
Huang J, Behrens C, Wistuba, II, Gazdar AF \& Jagirdar J 2002 Clonality of combined tumors. Archives of Pathology and Laboratory Medicine 126 437-441. (https://doi.org/10.1043/0003-

9985(2002)126<0437:COCT>2.0.CO;2)

Jesinghaus M, Konukiewitz B, Keller G, Kloor M, Steiger K, Reiche M, Penzel R, Endris V, Arsenic R, Hermann G, et al. 2017 Colorectal mixed adenoneuroendocrine carcinomas and neuroendocrine carcinomas are genetically closely related to colorectal adenocarcinomas. Modern Pathology 30 610-619. (https://doi org/10.1038/modpathol.2016.220)

Kim KM, Kim MJ, Cho BK, Choi SW \& Rhyu MG 2002 Genetic evidence for the multi-step progression of mixed glandular-neuroendocrine gastric carcinomas. Virchows Archiv 440 85-93. (https://doi. org/10.1007/s004280100540)

Kloppel G 2017 Neuroendocrine neoplasms: dichotomy, origin and classifications. Visceral Medicine 33 324-330. (https://doi. org/10.1159/000481390)

Konukiewitz B, Schlitter AM, Jesinghaus M, Pfister D, Steiger K, Segler A, Agaimy A, Sipos B, Zamboni G, Weichert W, et al. 2017 Somatostatin receptor expression related to TP53 and RB1 alterations in pancreatic and extrapancreatic neuroendocrine neoplasms with a Ki67-index above 20. Modern Pathology 30 587-598. (https://doi.org/10.1038/ modpathol.2016.217)

La Rosa S, Adsay V, Albarello L, Asioli S, Casnedi S, Franzi F, Marando A, Notohara K, Sessa F, Vanoli A, et al. 2012a Clinicopathologic study of 62 acinar cell carcinomas of the pancreas: insights into the morphology and immunophenotype and search for prognostic markers. American Journal of Surgical Pathology 36 1782-1795. (https://doi.org/10.1097/PAS.0b013e318263209d)

La Rosa S, Marando A, Furlan D, Sahnane N \& Capella C 2012b Colorectal poorly differentiated neuroendocrine carcinomas and mixed adenoneuroendocrine carcinomas: insights into the diagnostic immunophenotype, assessment of methylation profile, and search for prognostic markers. American Journal of Surgical Pathology 36 601-611. (https://doi.org/10.1097/PAS.0b013e318242e21c)

La Rosa S, Marando A, Sessa F \& Capella C 2012c Mixed adenoneuroendocrine carcinomas (MANECs) of the gastrointestinal tract: an update. Cancers 4 11-30. (https://doi.org/10.3390/ cancers4010011)

Lugli A, Kirsch R, Ajioka Y, Bosman F, Cathomas G, Dawson H, El Zimaity H, Flejou JF, Hansen TP, Hartmann A, et al. 2017 Recommendations for reporting tumor budding in colorectal cancer based on the International Tumor Budding Consensus Conference (ITBCC) 2016. Modern Pathology 30 1299-1311. (https://doi. org/10.1038/modpathol.2017.46)

Meazza C, Belfiore A, Busico A, Settanni G, Paielli N, Cesana L, Ferrari A, Chiaravalli S, Massimino M, Gronchi A, et al. 2016 AKT1 and BRAF mutations in pediatric aggressive fibromatosis. Cancer Medicine $\mathbf{5}$ 1204-1213. (https://doi.org/10.1002/cam4.669)

Milione M, Maisonneuve P, Spada F, Pellegrinelli A, Spaggiari P, Albarello L, Pisa E, Barberis M, Vanoli A, Buzzoni R, et al. 2017 The clinicopathologic heterogeneity of grade 3 gastroenteropancreatic neuroendocrine neoplasms: morphological differentiation and proliferation identify different prognostic categories. Neuroendocrinology 104 85-93. (https://doi.org/10.1159/000445165)

Ohike NA, La Rosa S, Volante M \& Zamboni G 2017 Mixed Neuroendocrinenon Neuroendocrine Neoplasms. Lyon, France: IARC Press.
Paniz Mondolfi AE, Slova D, Fan W, Attiyeh FF, Afthinos J, Reidy J, Pang Y \& Theise ND 2011 Mixed adenoneuroendocrine carcinoma (MANEC) of the gallbladder: a possible stem cell tumor? Pathology International 61 608-614. (https://doi. org/10.1111/j.1440-1827.2011.02709.x)

Rindi G, Arnold R, Bosman FT, Capella C, Klimstra DS, Klöppel G, Komminoth P \& Solcia E 2010 Nomenclature and Classification of Digestive Neuroendocrine Tumours. Eds TF Bosman, F Carneiro, RH Hruban \& ND Theise. Lyon, France: IARC Press.

Sahnane N, Furlan D, Monti M, Romualdi C, Vanoli A, Vicari E, Solcia E, Capella C, Sessa F \& La Rosa S 2015 Microsatellite unstable gastroinestinal neuroendocrin e carcinomas: a new clinicopathologic entity. Endocrine-Related Cancer 22 35-45. (https://doi.org/10.1530/ ERC-14-0410)

Scardoni M, Vittoria E, Volante M, Rusev B, Bersani S, Mafficini A, Gottardi M, Giandomenico V, Malleo G, Butturini G, et al. 2014 Mixed adenoneuroendocrine carcinomas of the gastrointestinal tract: targeted next-generation sequencing suggests a monoclonal origin of the two components. Neuroendocrinology 100 310-316. (https://doi. org/10.1159/000369071)

Shafqat H, Ali S, Salhab M \& Olszewski AJ 2015 Survival of patients with neuroendocrine carcinoma of the colon and rectum: a populationbased analysis. Diseases of the Colon and Rectum 58 294-303. (https:// doi.org/10.1097/DCR.0000000000000298)

Sorbye H, Welin S, Langer SW, Vestermark LW, Holt N, Osterlund P, Dueland S, Hofsli E, Guren MG, Ohrling K, et al. 2013 Predictive and prognostic factors for treatment and survival in 305 patients with advanced gastrointestinal neuroendocrine carcinoma (WHO G3): the NORDIC NEC study. Annals of Oncology 24 152-160. (https://doi. org/10.1093/annonc/mds276)

Volante M, Brizzi MP, Faggiano A, La Rosa S, Rapa I, Ferrero A, Mansueto G, Righi L, Garancini S, Capella C, et al. 2007 Somatostatin receptor type $2 \mathrm{~A}$ immunohistochemistry in neuroendocrine tumors: a proposal of scoring system correlated with somatostatin receptor scintigraphy. Modern Pathology 20 1172-1182. (https://doi.org/10.1038/modpathol.3800954)

Volante M, Monica V, Birocco N, Brizzi MP, Busso S, Daniele L, La Rosa S, Righi L, Sapino A, Berruti A, et al. 2015 Expression analysis of genes involved in DNA repair or synthesis in mixed neuroendocrine/nonneuroendocrine carcinomas. Neuroendocrinology 101 151-160. (https://doi. org/10.1159/000375449)

Vortmeyer AO, Lubensky IA, Merino MJ, Wang CY, Pham T, Furth EE \& Zhuang Z 1997 Concordance of genetic alterations in poorly differentiated colorectal neuroendocrine carcinomas and associated adenocarcinomas. Journal of the National Cancer Institute $\mathbf{8 9}$ 1448-1453. (https://doi.org/10.1093/jnci/89.19.1448)

Woischke C, Schaaf CW, Yang HM, Vieth M, Veits L, Geddert H, Markl B, Stommer P, Schaeffer DF, Frolich M, et al. 2017 In-depth mutational analyses of colorectal neuroendocrine carcinomas with adenoma or adenocarcinoma components. Modern Pathology 30 95-103. (https://doi.org/10.1038/modpathol.2016.150)

Xie JW, Lu J, Lin JX, Zheng CH, Li P, Wang JB, Chen QY, Cao LL, Lin M, Tu RH, et al. 2017 Different long-term oncologic outcomes after radical surgical resection for neuroendocrine carcinoma and adenocarcinoma of the stomach. Oncotarget 8 57495-57504. (https:// doi.org/10.18632/oncotarget.15488)

Received in final form 7 March 2018

Accepted 27 March 2018

Accepted Preprint published online 28 March 2018
2018 Society for Endocrinology Published by Bioscientifica Ltd. Printed in Great Britain 